\title{
MICROSTRUCTURE OF AN EQUIATOMIC CUPd ALLOY AFTER SEVERE PLASTIC DEFORMATION AND SUBSEQUENT ORDERING
}

\author{
O. V. Antonova, O. S. Novikova*, A. Yu. Volkov \\ M.N. Miheev Institute of Metal Physics of Ural Branch of Russian Academy of Sciences, 18 S. Kovalevskoy st., \\ Ekaterinburg, Russian Federation \\ *Corresponding author. E-mail: novikovav@imp.uran.ru, address for correspondence: 18, ul. S. Kovalevskoy, 620990, \\ Ekaterinburg, Russian Federation. Tel.: +7 343 3744054; fax: +7 3433745244 \\ The microstructure of an equiatomic CuPd alloy after severe plastic deformation (SPD) and \\ prolonged heat treatment in the ordering temperature range has been studied. A highly fragmented \\ nonuniform microstructure with high defect density is shown to be observed after SPD. After pro- \\ longed heat treatment, the bulk of the material is recrystallized and B2-type ordered (average grain \\ size is 0.3 to $0.35 \mu \mathrm{m})$.
}

Keywords: copper-palladium alloys, severe plastic deformation, phase transformations, microstructure.

DOI: $10.17804 / 2410-9908.2015 .5 .133-138$

\section{References}

1. Vandyshev A.B., Kulikov V.A. Calculating the main parameters of a membrane reformer with a production rate of $40 \mathrm{~m} 3 / \mathrm{h}$ designed for producing highly pure hydrogen from natural gas. Diagnostics, Resource and Mechanics of materials and structures. 2015, iss. 3, pp. 109-121. DOI: 10.17804/2410-9908.2015.3.109-121.

2. Burkhanov G.S., Gorina N.B., Kolchugina N.B., Roshan N.R. Palladium alloys for hydrogen power engineering. Rossiiskii Khimicheskii Zhurnal, 2006, vol. 50, no. 4, pp. 36-40. (In Russian).

3. Subramanian P.R., Laughlin D.E. Cu-Pd (Copper-Palladium). Journal of Phase Equilibria, 1991, vol. 12, issue 2, pp 231-243. DOI: 10.1007/BF02645723.

4. Volkov A.Yu, Novikova O.S., Antonov B.D. The kinetics of ordering in an equiatomic CuPd alloy: A resistometric study. Journal of Alloys and Compounds, 2013, vol. 581, pp. 625-631. DOI: 10.1016/j.jallcom.2013.07.132.

5. Kuranov A.A., Berseneva F.N., Sasinova R.A. Laptevskii A.S. Ordering and mechanical properties of Pd-Cu alloys. Fizika Metallov i Metallovedenie, 1983, vol. 56, no. 3, pp. 600-603. (In Russian).

6. Antonova O.V., Novikova O.S., Antonov B.D., Volkov A.Yu. Evolution of the Microstructure and Mechanical Properties of the $\mathrm{Cu}-47 \mathrm{Pd}$ (at \%) Alloy during Atomic Ordering after Severe Plastic Deformation. The Physics of Metals and Metallography, 2015, vol. 116, iss 4, pp. 401-412. DOI: $10.1134 / \mathrm{S} 0031918 X 1504002 X$.

7. Hirsch P.B., Howie A., Nicholson R.B., Pashley D.W., Whelan M.J. Electron Microscopy of Thin Crystals, London, Butterworths, 1965, 549 p.

8. Mishin O.V., Godfrey A., Juul Jensen D., Hansen N. Recovery and recrystallization in commercial purity aluminum cold rolled to an ultrahigh strain. Acta Materialia, 2013, vol. 61, iss. 14, pp. 5354-5364. DOI: 10.1016/j.actamat.2013.05.024. 
Подана в журнал: 10.09.2015

УДК 669.3'234:539.89:536.425

DOI: $10.17804 / 2410-9908.2015 .5 .133-138$

\title{
МИКРОСТРУКТУРА ЭКВИАТОМНОГО СПЛАВА СUРd ПОСЛЕ МЕГАПЛАСТИЧЕСКОЙ ДЕФОРМАЦИИ И ПОСЛЕДУЮЩЕГО УПОРЯДОЧЕНИЯ
}

\author{
О. В. Антонова, О. С. Новикова*, А. Ю. Волков \\ Федеральное государственное бюджетное учреждение науки Институт физики металлов имени \\ М.Н. Михеева Уральского отделения Российской академии наук, ул. С. Ковалевской, 18, Екатеринбург, \\ Российская Федерация \\ *Ответственный автор. Электронная почта: novikovav@imp.uran.ru, адрес для переписки: 620990, \\ ул. С. Ковалевской, 18, Екатеринбург, Россия. Телефон: +7 343 374-40-54; факс: +7 343 374-52-44
}

Проведено исследование микроструктуры эквиатомного сплава CuPd после мегапластической деформации (МПД) и после длительной термообработки в области температур упорядочения. Показано, что после МПД наблюдается микроструктура неоднородной морфологии, сильно фрагментированная и с высокой плотностью дефектов. После длительного отжига основной объем материала занимают рекристаллизованные зёрна размером $0,3-0,35$ мкм, упорядоченные по типу В2.

Ключевые слова: сплавы медь - палладий, мегапластическая деформачия, фазовые превращения, микроструктура.

\section{1. Введение}

Одним из актуальных направлений исследований в современных водородных технологиях является создание эффективных и экономичных систем для производства высокочистого водорода [1]. Сплавы медь - палладий вблизи эквиатомного состава являются перспективными материалами для водородной энергетики [2], так как практически не проявляют чувствительности к присутствию сероводорода, свойственную для чистого палладия, при одновременном снижении стоимости за счет большого содержания меди.

Согласно фазовой диаграмме [3], ниже температуры $510{ }^{\circ} \mathrm{C}$ сплав находится в двухфазной области (А1+В2) (А1 - неупорядоченная ГЦК-фаза; В2 - упорядоченная ОЦК-фаза).

Ранее нами было показано [4], что мегапластическая деформация (МПД) существенно ускоряет формирование упорядоченной структуры в эквиатомном сплаве CuPd. Рентгеноструктурный анализ сплава $\mathrm{CuPd}$ после МПД и последующего длительного отжига зафиксировал практически однофазное, упорядоченное по типу В2 состояние. В полученном структурном состоянии сплав имеет значение удельного электросопротивления $\rho=5,48 \times 10^{-8} \mathrm{Oм} \cdot \mathrm{M}$, что существенно ниже приведенных в литературе данных. Таким образом, использованный в [4] подход: длительный низкотемпературный отжиг после МПД впервые позволил сформировать в эквиатомном сплаве $\mathrm{CuPd}$ хорошо упорядоченное состояние. Именно в этом состоянии сплавы медь - палладий вблизи эквиатомного состава имеют перспективы использования в качестве мембран для сепарации водорода из газовых смесей.

В литературе отсутствуют микроструктурные исследования сплава $\mathrm{CuPd}$ после различных термомеханических обработок, за исключением [5]. Целью настоящей работы является проведение электронно-микроскопического исследования микроструктуры эквиатомного сплава CuPd после МПД прокаткой на высокие степени и последующих термообработок. 


\section{2. Материал и методика}

Сплав получали из меди и палладия чистотой 99,98 \% методом двойного переплава в вакууме не хуже $10^{-2}$ Па с разливкой в графитовый тигель. Полученный слиток диаметром 8 мм был гомогенизирован в течение 3 часов при температуре $850{ }^{\circ} \mathrm{C}$, а затем закален в воде. Состав сплава, согласно рентгеновскому микроанализу (Superprobe - JCXA - 733), соответствует следующему соотношению: $\mathrm{Cu}$ (49,8 ат. \%) - Pd (50,2 ат. \%). Слиток диаметром 8 мм подвергался прокатке до получения ленты толщиной 0,2 мм (истинная деформация е 23,8 ) без промежуточных отжигов.

Рентгеноструктурный анализ (PCA) осуществляли на дифрактометре DMAX 2200 фирмы Rigaku методом непрерывной съемки со скоростью 4 \% мин для съемки полной дифрактограммы и со скоростью 1 \%мин для выделенных участков. Излучение $\mathrm{CuK}_{\alpha}$ было монохроматизировано графитовым монокристаллом.

Исследование микроструктуры осуществляли с помощью просвечивающей электронной микроскопии на микроскопе JEM 200-CX при ускоряющем напряжении 160 кВ.

\section{3. Результаты и обсуждение}

Как отмечалось выше, сплав был подвергнут МПД от слитка до получения образцов требуемых размеров без промежуточных отжигов, при этом не наблюдалось каких-либо признаков разрушения. Ранее нами было установлено, что такая высокая пластичность сплавов $\mathrm{Cu}-(47-55)$ ат. \% Рd объясняется процессами динамического возврата [6].

Согласно данным РСА, эквиатомный сплав $\mathrm{CuPd}$ после МПД $(\mathrm{e} \approx 3,8)$ имеет однофазную ГЦК-структуру (A1). Рентгенограмма этого образца отличается наличием ярко выраженной текстуры (220). Параметр решетки сплава $\mathrm{CuPd}$, претерпевшего МПД, составляет $a=$ 0,3770 нм [4].

На рис. 1 а приведено наиболее типичное изображение микроструктуры после деформации е $\approx 3,8$. Видно, что на разных участках снимка наблюдается структура различной морфологии. В местах, указанных на рисунке стрелками, видны мелкие кристаллиты размерами $\leq 0,1$ мкм. Можно также различить слабые очертания деградированной полосовой структуры, например в левом углу рис. 1 a. На светлопольном изображении не фиксируются отчётливые границы от других структурных элементов. На микродифракции (МД) с центрального участка рис. 1 а наблюдается близкое к кольцевому расположение рефлексов (рис. 1 б), что указывает на большое количество элементов структуры разной ориентировки в выделенном объёме. Система колец отражения соответствует положению рефлексов, характерных для ГЦКрешетки (А1-фаза): первое кольцо - отражения от плоскостей типа $\{111\}$, второе - $\{200\}$, третье - $\{220\}$, четвёртое - $\{311\}$. На электронограмме видны также рефлексы, которым соответствует межплоскостное расстояние $\mathrm{d}=0,326$ нм (один из них отмечен символом $\square$ ). Они не принадлежат ни А1 (ГЦК), ни В2-фазе (ОЦК); в настоящей работе их природа не была выяснена. Более подробно проследить детали микроструктуры можно по темнопольным изображениям в группе рефлексов $\vec{g}=<111>$, один из которых выделен на МД символом (рис. 1 б). Рефлексы с азимутальной разориентировкой $\sim 3^{\circ}$ формируют контраст на полосе неправильной формы шириной приблизительно 0,35 мкм; также видны очертания полосы, расположенной почти перпендикулярно к первой (рис. 1 в). При этом существенное различие в интенсивности контраста указывает на фрагментацию внутри выделенных объёмов. Этот эффект отчётливо проступает при большем увеличении (рис. 1 2). Следует отметить, что в ходе исследования мы практически не наблюдали ни отдельных дислокаций, ни сложных дислокационных конфигураций. Контраст на электронно-микроскопических изображениях имеет много общего с картиной муара (указан стрелкой на рис. 1 2). Такое явление может быть вызвано малыми различиями межплоскостного расстояния по толщине фольги [7], которые возникают в результате искажений кристаллической решётки вследствие МПД [8], a также при концентрационных неоднородностях. 
opent-abeess jünal

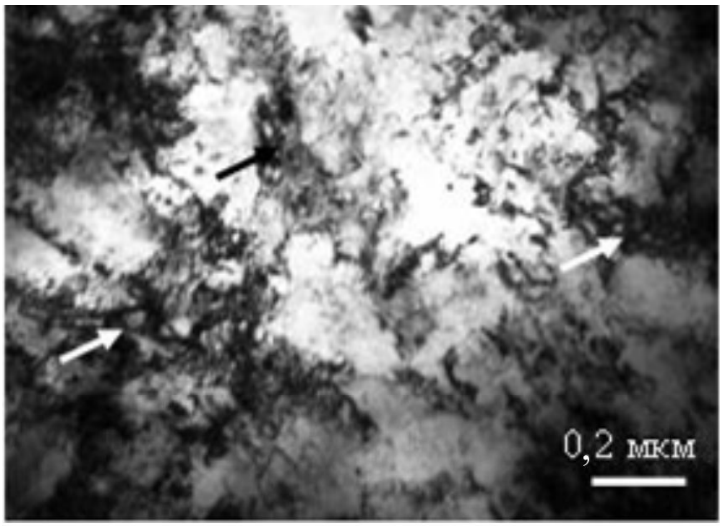

$a$

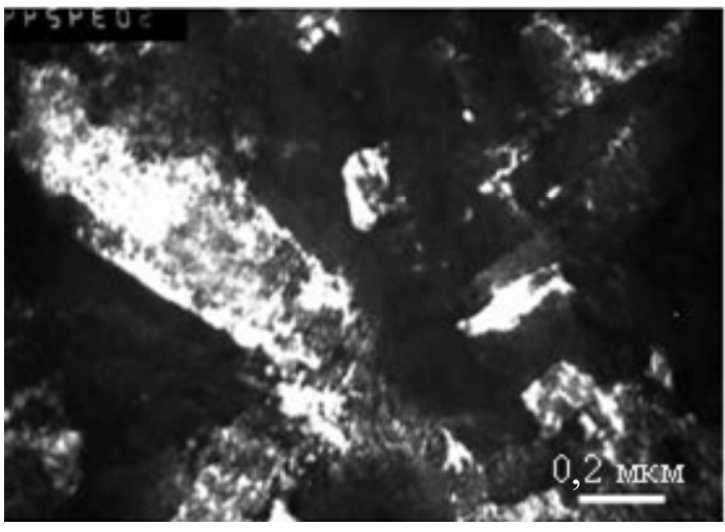

B

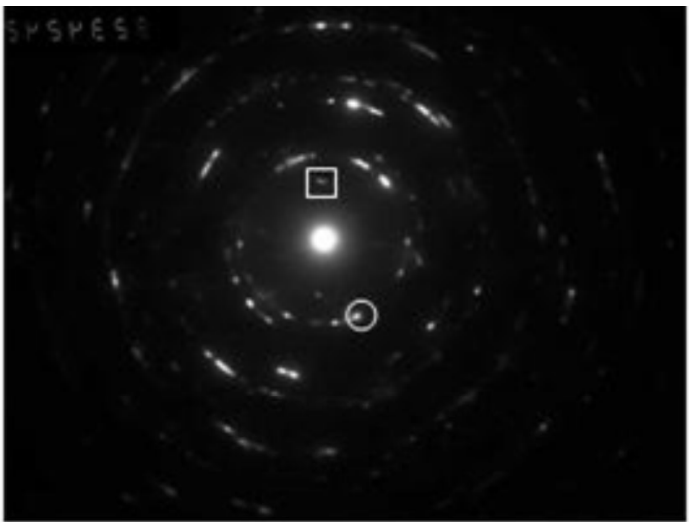

6

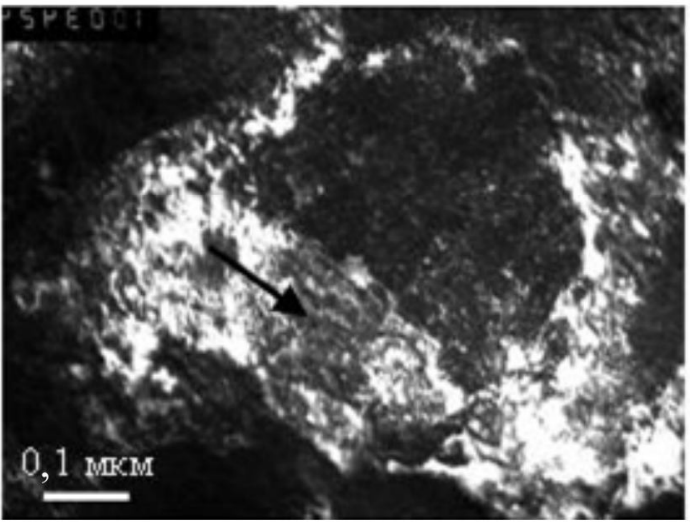

2

Рис. 1. Микроструктура эквиатомного сплава $\mathrm{CuPd}$ после МПД (е 23,8$): a$ - типичное светлопольное изображение; $\sigma$ - МД к $(a) ; \varepsilon$ - темнопольное изображение в группе рефлексов $\vec{g}$ $=<111>$; $2-$ центральный участок (в) при большем увеличении

Таким образом, получение тонких лент с помощью МПД не приводит к образованию трещин в материале, при этом позволяет сократить энергозатраты и количество операций при изготовлении мембран для альтернативной энергетики по сравнению с существующими технологиями.

Длительный ступенчатый отжиг сплава $\mathrm{CuPd}$ после МПД проводился по следующей схеме: охлаждение от $350{ }^{\circ} \mathrm{C}$ до $200{ }^{\circ} \mathrm{C}$ со скоростью 50 градусов в неделю с последующим охлаждением до комнатной температуры со скоростью 20 градусов в сутки. Согласно данным РСА [4], на дифрактограмме образца исследуемого сплава после такой обработки в явном виде присутствуют линии только В2-фазы. Степень дальнего атомного порядка, определённая по соотношению интенсивностей сверхструктурных и основных линий, соответствует хорошо упорядоченному состоянию $(\eta \approx 0,97)$. Параметр упорядоченной решетки сплава: $a=$ 0,2978 HM. 


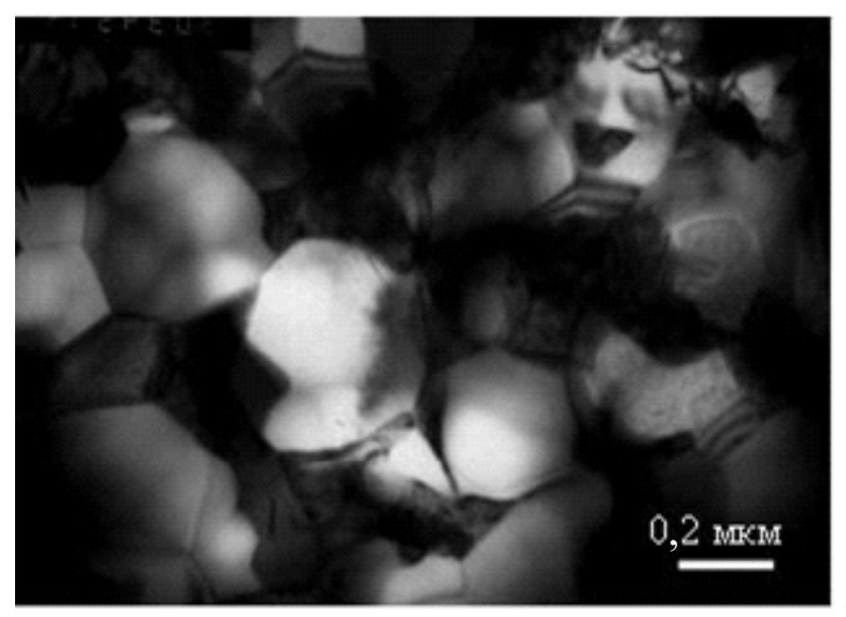

$a$

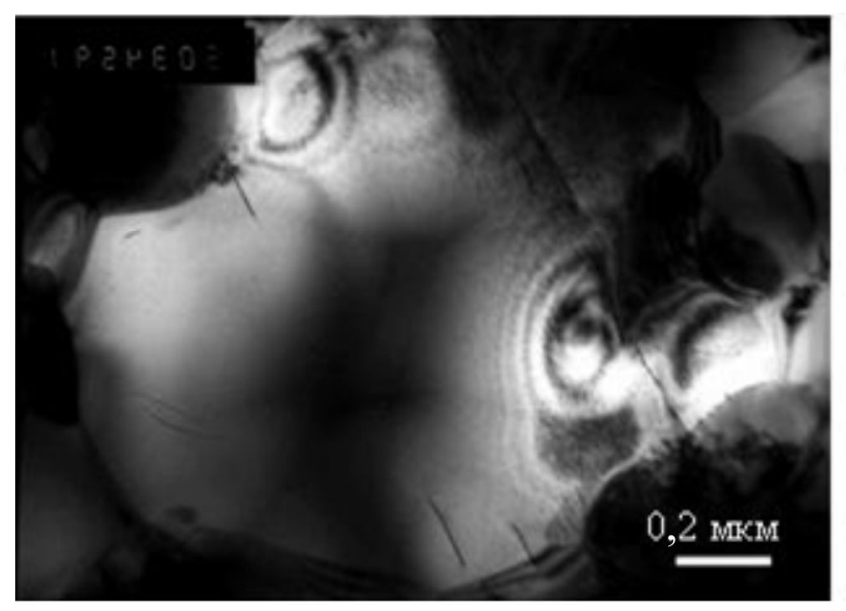

B

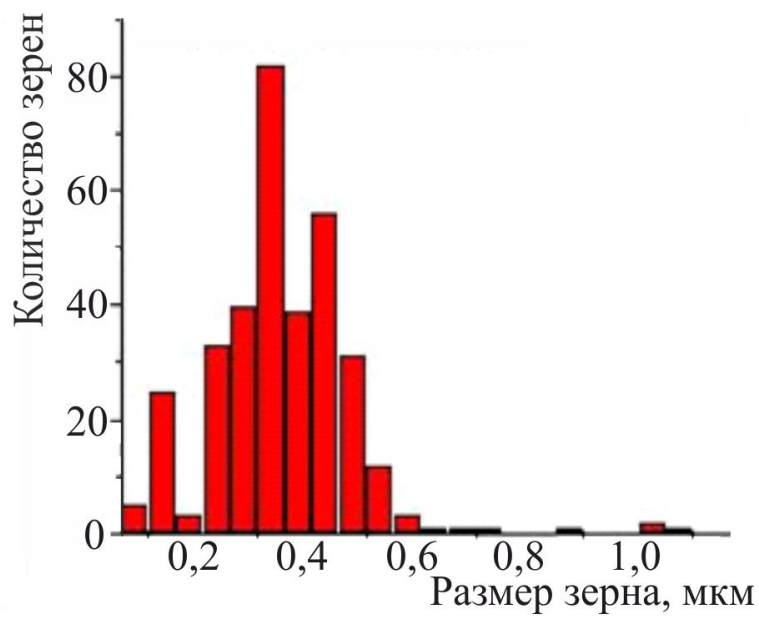

$\sigma$

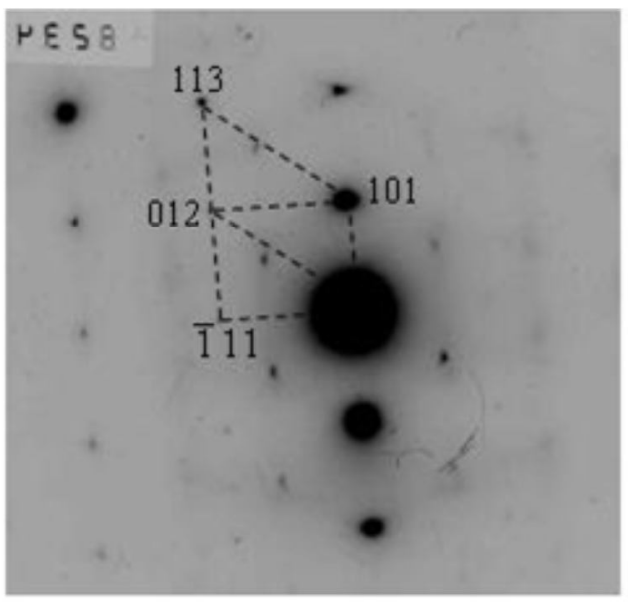

2

Рис. 2. Микроструктура упорядоченного сплава $\mathrm{CuPd}$, после МПД (e $\approx 3,8)$ и отжига: $a$ - типичное светлопольное изображение; $\sigma$ - гистограмма распределения зёрен по размеру (на основе обработки 300 зёрен); в - пример развития вторичной рекристаллизации;

$$
\text { г-МД к (в) }
$$

Электронно-микроскопические исследования подтвердили данные РСА: практически весь объём материала представлен новыми, упорядоченными по типу В2 зёрнами (рис. $2 a$ ). Наблюдается рекристаллизованная структура с размером зерна 0,3-0,35 мкм (рис. 2 б). Среди равноосных, однородно распределённых по размерам зёрен встречаются отдельные укрупнённые зёрна (рис. 2 в), что может свидетельствовать о вторичной рекристаллизации. МД с крупного зерна (рис. 2 г) является сечением обратной решётки с осью зоны [1 $\overline{2} 1]$. Анализ полученных результатов позволяет предположить, что при упорядочении сплава $\mathrm{CuPd}$ после МПД скорости конкурирующих процессов (рекристаллизации и фазового превращения $\mathrm{A} 1 \rightarrow \mathrm{B} 2)$, сопоставимы. Иерархия этих процессов должна быть исследована более подробно.

Таким образом, длительный отжиг сплава приводит к получению хорошо упорядоченного состояния. Тонкие фольги из сплава $\mathrm{CuPd}$ в таком состоянии являются перспективным материалом для водородной энергетики. В дальнейшем предполагается проведение подробных экспериментов по изучению их водородопроницаемости. 


\section{4. Заключение}

1. В сплаве $\mathrm{CuPd}$ после МПД прокаткой $(\mathrm{e} \approx 3,8)$ формируется неоднородная по объёму, фрагментированная микроструктура с высокой плотностью дефектов и отдельными кристаллитами размером меньше 0,1 мкм.

2. В результате длительного отжига сплава $\mathrm{CuPd}$ после МПД наблюдается рекристаллизованная, хорошо упорядоченная по типу В2 структура со средним размером зерна $0,3-0,35$ мкм.

\section{Благодарность}

Электронно-микроскопические исследования проведены в ОЭМ ЦКП ИФМ УрО РАН. Работа выполнена в рамках государственного задания ФАНО России (тема «Деформация», № 01201463327) и при частичной поддержке проекта УрО РАН № 15-17-2-11.

\section{Литература}

1. Vandyshev A. B., Kulikov V. A. Calculating the main parameters of a membrane reformer with a production rate of $40 \mathrm{~m} 3 / \mathrm{h}$ designed for producing highly pure hydrogen from natural gas // Diagnostics, Resource and Mechanics of materials and structures. - 2015. - Iss. 3. - P. 109-121.

2. Сплавы палладия для водородной энергетики / Г. С. Бурханов, Н. Б. Горина, Н. Б. Кольчугина, Н. Р. Рошан // Рос. хим. журнал (Журнал рос. хим. об-ва им. Д.И. Менделеева). - 2006. - Т. 50, № 4. - С. 36-40.

3. Subramanian P. R., Laughlin D. E. Cu-Pd (Copper-Palladium) // Journal of Phase Equilibria. - 1991. - Vol. 12, iss. 2. - P. 231-243. - DOI: 10.1007/BF02645723.

4. Volkov A. Yu, Novikova O. S., Antonov B. D. The kinetics of ordering in an equiatomic CuPd alloy: A resistometric study // J. Alloys Compd. - 2013. - Vol. 581. - P. 625-631.

5. Упорядочение и механические свойства сплавов палладий-медь / А. А. Куранов, Ф. Н. Берсенева, Р. А. Сасинова, А. С. Лаптевский // ФММ. - 1983. - Т. 56, № 3. - С. 600-603. 6. Evolution of the Microstructure and Mechanical Properties of the $\mathrm{Cu}-47 \mathrm{Pd}$ (at \%) Alloy during Atomic Ordering after Severe Plastic Deformation / O. V. Antonova, O. S. Novikova, B. D. Antonov, A. Yu. Volkov // The Physics of Metals and Metallography. - 2015. - Vol. 116, iss 4. - P. 401-412. - DOI: 10.1134/S0031918X1504002X.

7. Электронная микроскопия тонких кристаллов / П. Хирш, А. Хови, Р. Николсон, Д. Пэшли, М. Уэлан / Пер. с англ. под ред. Л. М. Утевского. - М.: Мир, 1968. - 575 с.

8. Recovery and recrystallization in commercial purity aluminum cold rolled to an ultrahigh strain / O. V. Mishin, A. Godfrey, D. Juul Jensen, N. Hansen // Acta Materialia - 2013. - Vol. 61, iss. 14. - P. 5354-5364. - DOI: 10.1016/j.actamat.2013.05.024. 